\title{
Comparison of Outcomes in Obese Patients After Total Knee Arthroplasty With Neutral or Mild Varus: a Retrospective Study With 8-year Follow-up
}

Ya-hao Lai

Sichuan University West China Hospital

Jian Cao

Sichuan University West China Hospital

Wei Feng

Sichuan University West China Hospital

Ze-xi Li

Sichuan University West China Hospital

Hong Xu

Sichuan University West China Hospital

Zongke Zhou ( $\square$ zhouzongke@scu.edu.cn )

Sichuan University West China Hospital https://orcid.org/0000-0002-9037-4756

\section{Research Article}

Keywords: Obesity, Varus, Neutral, Alignment, Total knee arthroplasty

Posted Date: December 13th, 2021

DOI: https://doi.org/10.21203/rs.3.rs-1154370/v1

License: () (1) This work is licensed under a Creative Commons Attribution 4.0 International License.

Read Full License 


\section{Abstract}

Background Residual varus after total knee arthroplasty (TKA) can affect functional outcomes and prosthetic survival, which may worsen in the presence of obesity. However, no studies were found to compare the outcomes of obese patients involving postoperative residual mild varus or neutral. The aim of this study was to compare outcomes for knees of obese patients with neutral or mild varus after TKA.

Methods We retrospectively reviewed 188 consecutive obese patients (body mass index $\geq 30 \mathrm{~kg} / \mathrm{m}^{2}$ ) at our hospital who underwent TKA due to varus knee osteoarthritis from January 2010 to December 2015. The mechanical hip-knee-ankle axis angle was measured in all patients pre- and postoperatively. Prosthesis survival and knee function were retrospectively assessed based on the Western Ontario and McMaster Universities Osteoarthritis Index (WOMAC) score, Knee Society Knee Score (KS-KS), Knee Society Function Score (KS-FS), Forgotten Joint Score (FJS), and range of motion (ROM).

Results Of the 156 knees in 132 obese patients who completed follow-up for a mean of $8.32 \pm 1.47$ years, 97 knees were corrected from varus to neutral and 54 knees were kept in mild residual varus. Knees with mild varus showed significantly better WOMAC and FJS scores. The two types of knees did not differ significantly in KS-KS, KS-FS or ROM. Although one patient with a neutral knee had to undergo revision surgery, there was no significant difference between two groups.

Conclusions For obese patients with osteoarthritis, preservation of residual varus alignment after TKA can improve functional outcomes without compromising prosthesis survival.

\section{Introduction}

Since 1980, the prevalence of overweight and obesity has increased to the point that nearly a third of the world's population is overweight or obese ${ }^{[1]}$. Obesity is a risk factor for osteoarthritis ${ }^{[2]}$, so the proportion of patients undergoing total knee arthroplasty (TKA) who are obese is expected to increase ${ }^{[3]}$. Obesity can lead to osteoarthritis that requires surgical treatment at an earlier age than in non-obese individuals ${ }^{[4]}$, and obesity can increase the risk of perioperative and long-term complications ${ }^{[5,6]}$. It may also increase risk of poor outcome and prosthesis failure after TKA ${ }^{[7]}$.

Some work has suggested that neutral mechanical alignment after TKA, defined as an adjustment within $3^{\circ}$ of neutral ${ }^{[8]}$, may prolong prosthesis survival and improve function ${ }^{[9,10]}$. However, other studies have failed to detect a significant difference in survivorship ${ }^{[1-13]}$ or other outcomes ${ }^{[14]}$ after TKA in knees with neutral or mild varus alignment. Thus, further study is needed to clarify whether neutral alignment is beneficial for TKA patients. In particular, whether it benefits obese patients should be addressed, since neutral alignment is more difficult to achieve in these patients ${ }^{[15]}$. In addition, obese patients may be at higher risk of aseptic tibial loosening regardless of coronal alignment ${ }^{[16]}$, since the excessive body weight is transferred to surrounding bone $\mathrm{e}^{[17]}$. 
The present study assessed the potential benefits of neutral or mild varus alignment in obese patients after TKA. We retrospectively compared functional outcomes and prosthesis survival during mean followup of 8 years.

\section{Methods}

\section{Patients}

We retrospectively screened all patients at our hospital who underwent primary TKA for varus-type knee osteoarthritis from January 2010 to December 2015. During this period, we performed 701 cemented TKAs in 663 patients, 188 of whom (216 knees) were obese, defined as a body mass index $\geq 30 \mathrm{~kg} / \mathrm{m}^{2}$ [18].

From the 188 obese patients, we excluded two who achieved normal weight at our follow-up, eight who had valgus or neutral alignment before TKA, two with a history of hip arthroplasty or extra-articular deformity that could affect ipsilateral limb alignment, and 44 who were lost to follow-up. In the end, we included 132 patients (156 knees).

The mechanical hip-knee-ankle axis angle (HKA) was measured in all patients before and after TKA based on full-length radiography conducted while patients were standing and the patella was oriented forward. Angles were determined independently by two investigators.

\section{Surgical technique}

All procedures were performed by one surgeon, who performed a midline skin incision and employed a medial parapatellar approach. After retracing the patella, the anterior cruciate ligaments and meniscus were completely resected. Osteotomy was performed using an intramedullary jig for the distal femur and extramedullary jig for the proximal tibia, and the valgus angle during osteotomy was determined according to preoperative full-length radiographs to achieve neutral alignment.

On the femoral side Whiteside's line and the anatomical transepicondylar axis were used as landmarks to position the intramedullary guides, while on the tibial side, the center of the tibial intercondylar eminence and the center of the ankle were used as landmarks to position the extramedullary guides. The tibia was cut, the posterior cruciate ligament was resected, and soft tissue was released according to the degree of preoperative flexion contraction. Depending on the patient's degree of varus, polyethylene spacers of varying thickness were inserted, or additional soft tissue was released. All patients received a cemented total knee prosthesis and underwent patelloplasty without patellar resurfacing.

\section{Data collection}


Body mass index was calculated as weight $(\mathrm{kg})$ divided by the square of the height $(\mathrm{m})$. HKA was expressed as a deviation from $180^{\circ}$, with positive values indicating valgus and negative values indicating varus alignment. Based on postoperative HKA, knee alignment was classified as valgus $\left(H K A>3^{\circ}\right)$, neutral $\left(-3^{\circ} \leq \mathrm{HKA} \leq 3^{\circ}\right)$, mild varus $\left(-6^{\circ} \leq \mathrm{HKA}<-3^{\circ}\right)$, or severe varus $\left(\mathrm{HKA}<-6^{\circ}\right)$. Most comparisons in this study were performed between knees showing neutral or mild varus alignment.

\section{Follow-up}

Patients were followed up by outpatient visit. At follow-up, we measured range of motion (ROM), Western Ontario and McMaster Universities Osteoarthritis Index score (WOMAC), Keen Society Score (KSS), and Forgotten Joint Score (FJS). Prosthesis infection and aseptic prosthesis loosening were recorded to assess prosthesis survival.

\section{Statistical analysis}

All analyses were performed in SPSS 26.0 (IBM, Armonk, NY, USA). Normally distributed data were expressed as mean \pm standard deviation (SD), otherwise data were expressed as median (25th percentile, 75th percentile). Continuous data such as age, follow-up duration, HKA, ROM, WOMAC, KSS, and FJS were compared between knees with neutral or mild varus alignment using analysis of Student's $t$ test or variance or the Kruskal-Wallis test as appropriate. Categorical data were compared using the chi-squared test. Differences associated with $\mathrm{P}<0.05$ were considered significant.

\section{Results}

Of the 156 knees in 132 obese patients who completed follow-up, 97 knees were corrected from varus to neutral, while 54 remained in mild varus. Age, sex, body mass index, preoperative HKA, and follow-up duration did not differ significantly between patients whose knees had neutral alignment and those whose knees had mild varus (Table 1). Neutral knees were corrected from a preoperative mean of $-8.7^{\circ}$ to $-1.0^{\circ}$ (Table 2). Mild varus knees were corrected from a preoperative mean of $-9.8^{\circ}$ to $-4.3^{\circ}$. 
Table 1

Characteristics of patients stratified according to whether their knees showed neutral or mild residual varus alignment after TKA

\begin{tabular}{|llll|}
\hline Characteristic & $\begin{array}{l}\text { Neutral } \\
\text { (n=97 knees) }\end{array}$ & $\begin{array}{l}\text { Mild varus } \\
\text { (n=54 knees) }\end{array}$ & P * \\
\hline Patients & 86 & 46 & 0.174 \\
Sex & 13 & 5 & \\
Male & 73 & 41 & 0.921 \\
Female & $72.84(6.664)$ & $74.39(6.700)$ & 0.964 \\
Age (years) & $31.49(1.355)$ & $31.47(1.441)$ & 0.255 \\
Body mass index $\left(\mathrm{kg} / \mathrm{m}^{2}\right)$ & $-8.7(4.962)$ & $-9.8(4.374)$ & 0.060 \\
Preoperative HKA & $8.13(1.455)$ & $8.62(1.538)$ & \\
Follow-up (years) & & \\
Values are $n$ or mean $(S D)$ unless otherwise noted. & & \\
HKA: hip-knee-ankle axis angle; TKA: total knee arthroplasty & & \\
* Based on Student's $t$ test or Mann-Whitney test (continuous variables) or chi-squared test & \\
\hline (categorical variables) & & \\
\hline
\end{tabular}

Table 2

Hip-knee-ankle axis angle before and after TKA for knees stratified based on postoperative alignment

\begin{tabular}{|llll|}
\hline Alignment & $\mathbf{n}$ (knees) & Preoperative & Postoperative \\
\hline Neutral & 97 & $-8.7(4.962)$ & $-1.0(0.129)$ \\
Mild varus & 54 & $-9.8(4.374)$ & $-4.3(0.127)$ \\
Severe varus & 1 & -16.4 & -7.3 \\
Valgus & 4 & $-3.51(3.025)$ & $3.84(0.336)$ \\
\hline \multicolumn{2}{l}{ Values are $n$ or mean (standard deviation). } \\
\hline
\end{tabular}

Patients whose knees had neutral or mild varus did not differ significantly in postoperative ROM, KS-KS, or KS-FS (Table 3). Patients with mild varus knees had significantly better WOMAC (8.25 \pm 8.637 vs 14.97 $\pm 14.193, \mathrm{P}=0.048$; Figure 1$)$ and better FJS $(86.03 \pm 15.607$ vs $70.22 \pm 30.031, \mathrm{P}=0.021$; Figure 2$)$. 
Table 3

Functional outcomes and prosthesis survival after TKA for knees stratified by postoperative alignment

\begin{tabular}{|llll|}
\hline Score or outcome & $\begin{array}{l}\text { Neutral } \\
\text { (n=97 knees) }\end{array}$ & $\begin{array}{l}\text { Mild varus } \\
\text { (n=54 knees) }\end{array}$ & P * \\
\hline Range of motion $\left(^{\circ}\right)$ & $92.34(17.320)$ & $100.00(13.633)$ & 0.130 \\
WOMAC score & $14.97(14.193)$ & $8.25(8.637)$ & 0.048 \\
Knee Society knee score & $83.66(10.640)$ & $87.25(8.095)$ & 0.201 \\
Knee Society function score & $75.69(20.139)$ & $78.31(17.366)$ & 0.643 \\
Forgotten joint score & $70.22(30.031)$ & $86.03(15.607)$ & 0.021 \\
\hline Revision & 1 & 0 & 1.000 \\
\hline Values are $n$ or mean (standard deviation) unless otherwise noted. & \\
$\begin{array}{l}\text { TKA, Total knee arthroplasty; WOMAC: Western Ontario and McMaster Universities Osteoarthritis } \\
\text { Index score }\end{array}$ & \\
* Based on Student's t test or Mann-Whitney test (continuous variables) or chi-squared test \\
(categorical variables)
\end{tabular}

One patient with a neutral knee had to undergo revision surgery due to aseptic loosening of the tibial component.

\section{Discussion}

Our retrospective study suggests that there is no significant difference in prosthesis survival between obese patients who have neutral or mild residual varus after TKA. Nevertheless, we found evidence that mild postoperative varus alignment is associated with better outcomes than neutral alignment.

Traditionally, optimal outcomes in TKA have been thought to depend on achieving a lower-extremity HKA within $3^{\circ}$ of neutral and positioning tibial and femoral components perpendicular to the mechanical axis in the coronal plane ${ }^{[19-21]}$. On the other hand, several studies ${ }^{[21-23]}$ have questioned this long-held tenet, arguing that it creates an unnatural situation for at least a subset of patients. Assessing the validity of this practice may be particularly important for obese patients, whose knees are overloaded and in whom the load is distributed onto the tibial component ${ }^{[7,24]}$. Previous studies ${ }^{[11,14,23,25-32]}$ comparing outcomes between TKA patients with postoperative neutral or mild varus did not distinguish between obese and non-obese patients.

Our finding that postoperative neutral or mild varus did not significantly affect prosthesis survival for obese patients after TKA. Similar results were found from several studies ${ }^{[11,25-27]}$ that did not distinguish obese from non-obese patients. However, it contrasts with the conclusion of a meta-analysis ${ }^{[33]}$ of 10 
studies involving 12,278 knees, according to which mild or severe residual varus shortens prosthesis survival. Instead, those investigators concluded that neutral or valgus alignment is essential for long-term prosthesis survival. It is difficult to compare that meta-analysis with our study because they did not distinguish between mild and severe varus. Our results also contrast with a study ${ }^{[28]}$ in which left $3^{\circ}$ varus alignment was linked to adduction overload of the medial compartment, causing asymmetrical polyethylene liner wear, tibial component loosening and mechanical failure. Our finding should be verified and extended in studies with even longer follow-up, given the length of time needed to adequately assess outcomes; and in studies involving morbidly obese patients, who were rare in our sample. Such patients may be at even higher risk of asymmetric polyethylene lining wear, loosening of tibial components, and mechanical failure ${ }^{[2]}$.

Our study suggests that mild postoperative varus alignment led to better functional outcomes than neutral alignment. Our results are similar to those of three studies ${ }^{[25,31,32]}$ reporting better outcomes for mild varus alignment after mean follow-up of 4-7 years, but our results contrast with those of four studies $^{[14,23,29,30]}$ that failed to find a significant difference in outcomes for mean follow-up for up to five years. One study ${ }^{[34]}$ has suggested that mild residual varus alignment can create physiological tension in the soft tissue that feels more "natural" to patients, and we speculate that this benefit may increase with time, which may explain why mainly studies with longer follow-up have detected it. Our finding may be especially important not only because of our long follow-up but also because our assessment included the FJS, which measures patients' ability to forget the artificial joint in everyday life. This index is likely to be strongly associated with patient satisfaction ${ }^{[35]}$. In recent years, kinematically aligned (KA) TKA was developed to restore normal knee function by maintaining the soft tissue envelope and minimizing the need for ligament release ${ }^{[36-38]}$. However, mechanical alignment (MA) TKA may demand higher release of soft tissues and ligaments, especially in patients with severe varus, which contrasts with KA TKA concepts. Our results that it is not that important to merely generate a neutral HKA angle, lend support to the emerging view that TKA patients can benefit more from KA TKA than from MA TKA ${ }^{[39-41]}$.

Our findings should be interpreted with caution in light of several limitations. First, our finding of no significant difference in prosthesis survival should be considered preliminary until it can be verified in larger samples with even longer follow-up. Second, we included only patients with primary knee osteoarthritis, which led to the same prominant female bias in our sample as in most other studies similar to ours ${ }^{[42]}$. Third, we did not closely examine patients with severe preoperative varus, who require further study because soft tissue release is more challenging in such cases. Similarly, further study is needed that differentiates obese patients by the severity of their obesity and comorbidities.

\section{Conclusion}

Our relatively small, retrospective study suggests that retaining slight varus in the knees of obese patients undergoing TKA may lead to better functional outcomes without reducing prosthesis survival, even in the long term. 


\section{Abbreviations}

TKA: Total knee arthroplasty

WOMAC: Western Ontario and McMaster Universities Osteoarthritis Index score

KS-KS: Knee Society Knee Score

KS-FS: Knee Society Function Score

FJS: Forgotten Joint Score

ROM: Range of motion

HKA: Hip-knee-ankle axis angle

SD: standard deviation

BMI: Body mass index

KA: Kinematically aligned

MA: Mechanical alignment

\section{Declarations}

\section{Ethics approval and consent to participate}

The hospital's institutional review board approved the study. Written informed consent was deemed unnecessary by the hospital's institutional review board.

\section{Consent for publication}

All authors have stated for consents of publications. Availability of data and materials. The data sets used and/or analyzed during the current study are fully available on reasonable request.

\section{Competing interest}

The authors have no relevant financial or non-financial interests to disclose. Funding This work was supported by 1.3.5 project for disciplines of excellence, West China Hospital, Sichuan University and Regional Innovation \& Cooperation program of Science \& Technology Department of Sichuan Province (No. 2021YFQ0028).

\section{Authors' contributions}


Yahao Lai and Jian Cao carried out the concepts, design, definition of intellectual content, data acquisition, data analysis and manuscript preparation. Wei Feng, Mengxi Li and Hong Xu provided assistance for data acquisition, data analysis and statistical analysis. Zongke Zhou completed all operations and helped to performed manuscript review. All authors reviewed the final manuscript. All authors agree to be accountable for all aspects of the work.

\section{Acknowledgements}

Not applicable

\section{Code availability}

Not applicable

\section{Publisher's Note}

Springer Nature remains neutral with regard to jurisdictional claims in published maps and institutional affiliations.

\section{References}

1. Murray CJL, Kyu HH, Maddison ER, et al. The global burden of tuberculosis: results from the Global Burden of Disease Study 2015'. Lancet Infectious Diseases. 2018;18(3):261-84.

2. Boyce L, Prasad A, Barrett M, et al. The outcomes of total knee arthroplasty in morbidly obese patients: a systematic review of the literature. Archives of Orthopaedic and Trauma Surgery. 2019;139(4):553-60.

3. Johnson CA, White CC, Kunkle BF, Eichinger JK, Friedman RJ. Effects of the Obesity Epidemic on Total Hip and Knee Arthroplasty Demographics. Journal of Arthroplasty. 2021;36(9):3097-100.

4. Lee R, Kean WF. Obesity and knee osteoarthritis. Inflammopharmacology. 2012;20(2):53-8.

5. Yeung E, Jackson M, Sexton S, Walter W, Zicat B, Walter W. The effect of obesity on the outcome of hip and knee arthroplasty. International Orthopaedics. 2011;35(6):929-34.

6. McElroy MJ, Pivec R, Issa K, Harwin SF, Mont MA. The Effects of Obesity and Morbid Obesity on Outcomes in TKA. Journal of Knee Surgery. 2013;26(2):83-8.

7. Gunst S, Fessy M-H. The effect of obesity on mechanical failure after total knee arthroplasty. Annals of Translational Medicine. 2015;3(20).

8. Fang DM, Ritter MA, Davis KE. Coronal Alignment in Total Knee Arthroplasty Just How Important is it? Journal of Arthroplasty. 2009;24(6):39-43.

9. Abdel MP, Oussedik S, Parratte S, Lustig S, Haddad FS. Coronal alignment in total knee replacement HISTORICAL REVIEW, CONTEMPORARY ANALYSIS, AND FUTURE DIRECTION. Bone \& Joint Journal. 2014;96B(7):857-62. 
10. Hungerford DS, editor Alignment in total knee replacement. 1994 Instructional Course Lectures, at the 61st Annual Meeting of the American-Academy-of-Orthopaedic-Surgeons; 1995 1994; New Orleans, La1995.

11. Parratte S, Pagnano MW, Trousdale RT, Berry DJ. Effect of Postoperative Mechanical Axis Alignment on the Fifteen-Year Survival of Modern, Cemented Total Knee Replacements. Journal of Bone and Joint Surgery-American Volume. 2010;92A(12):2143-9.

12. Morgan SS, Bonshahi A, Pradhan N, Gregory A, Gambhir A, Porter ML. The influence of postoperative coronal alignment on revision surgery in total knee arthroplasty. International Orthopaedics. 2008;32(5):639-42.

13. Dossett HG, Swartz GJ, Estrada NA, LeFevre GW, Kwasman BG. Kinematically Versus Mechanically Aligned Total Knee Arthroplasty. Orthopedics. 2012;35(2):E160-E9.

14. Zhang Z, Liu C, Li Z, Wu P, Hu S, Liao W. Residual Mild Varus Alignment and Neutral Mechanical Alignment Have Similar Outcome after Total Knee Arthroplasty for Varus Osteoarthritis in Five-Year Follow-Up. Journal of Knee Surgery. 2020;33(2):200-5.

15. Jarvenpaa J, Kettunen J, Kroger H, Miettinen H. OBESITY MAY IMPAIR THE EARLY OUTCOME OF TOTAL KNEE ARTHROPLASTY A prospective study of 100 patients. Scandinavian Journal of Surgery. 2010;99(1):45-9.

16. Abdel MP, Bonadurer GF, III, Jennings MT, Hanssen AD. Increased Aseptic Tibial Failures in Patients With a BMI >= 35 and Well-Aligned Total Knee Arthroplasties. Journal of Arthroplasty. 2015;30(12):2181-4.

17. Morrison JB. MECHANICS OF KNEE JOINT IN RELATION TO NORMAL WALKING. Journal of Biomechanics. 1970;3(1):51-\&.

18. Consultation WHO, Who WHO. Obesity: Preventing and managing the global epidemic - Introduction. Obesity: Preventing and Managing the Global Epidemic: Report of a Who Consultation. WHO Technical Report Series. 8942000. p. 1-253.

19. Berend ME, Ritter MA, Meding JB, et al. Tibial component failure mechanisms in total knee arthroplasty. Clinical Orthopaedics and Related Research. 2004(428):26-34.

20. Choong PF, Dowsey MM, Stoney JD. Does Accurate Anatomical Alignment Result in Better Function and Quality of Life? Comparing Conventional and Computer-Assisted Total Knee Arthroplasty. Journal of Arthroplasty. 2009;24(4):560-9.

21. Howell SM, Papadopoulos S, Kuznik KT, Hull ML. Accurate alignment and high function after kinematically aligned TKA performed with generic instruments. Knee Surgery Sports Traumatology Arthroscopy. 2013;21(10):2271-80.

22. Bellemans J, Colyn W, Vandenneucker H, Victor J. The Chitranjan Ranawat Award: Is Neutral Mechanical Alignment Normal for All Patients?: The Concept of Constitutional Varus. Clinical Orthopaedics and Related Research. 2012;470(1):45-53.

23. Rames RD, Mathison M, Meyer Z, Barrack RL, Nam D. No impact of under-correction and joint line obliquity on clinical outcomes of total knee arthroplasty for the varus knee. Knee Surgery Sports 
Traumatology Arthroscopy. 2018;26(5):1506-14.

24. Amin AK, Clayton RAE, Patton JT, Gaston M, Cook RE, Brenkel IJ. Total knee replacement in morbidly obese patients - Results of a prospective, matched study. Journal of Bone and Joint Surgery-British Volume. 2006;88B(10):1321-6.

25. Nishida K, Matsumoto T, Takayama K, et al. Remaining mild varus limb alignment leads to better clinical outcome in total knee arthroplasty for varus osteoarthritis. Knee Surgery Sports Traumatology Arthroscopy. 2017;25(11):3488-94.

26. Salzmann M, Fennema P, Becker R, Hommel H. Does Postoperative Mechanical Axis Alignment Have an Effect on Clinical Outcome of Primary Total Knee Arthroplasty? A Retrospective Cohort Study. The open orthopaedics journal. 2017;11:1330-6.

27. Bonner TJ, Eardley WGP, Patterson P, Gregg PJ. The effect of post-operative mechanical axis alignment on the survival of primary total knee replacements after a follow-up of 15 years. Journal of Bone and Joint Surgery-British Volume. 2011;93B(9):1217-22.

28. Howell SM, Papadopoulos S, Kuznik K, Ghaly LR, Hull ML. Does varus alignment adversely affect implant survival and function six years after kinematically aligned total knee arthroplasty? International Orthopaedics. 2015;39(11):2117-24.

29. Magnussen RA, Weppe F, Demey G, Servien E, Lustig S. Residual Varus Alignment does not Compromise Results of TKAs in Patients with Preoperative Varus. Clinical Orthopaedics and Related Research. 2011;469(12):3443-50.

30. Hatayama K, Terauchi M, Saito K, Higuchi H. Does Residual Varus Alignment Cause Increasing Varus Laxity at a Minimum of Five Years After Total Knee Arthroplasty? Journal of Arthroplasty. 2017;32(6):1808-13.

31. Vanlommel L, Vanlommel J, Claes S, Bellemans J. Slight undercorrection following total knee arthroplasty results in superior clinical outcomes in varus knees. Knee Surgery Sports Traumatology Arthroscopy. 2013;21(10):2325-30.

32. Schiffner E, Wild M, Regenbrecht B, et al. Neutral or Natural? Functional Impact of the Coronal Alignment in Total Knee Arthroplasty. Journal of Knee Surgery. 2019;32(8):820-4.

33. Liu $H-x$, Shang $P$, Ying $X-Z$, Zhang Y. Shorter survival rate in varus-aligned knees after total knee arthroplasty. Knee Surgery Sports Traumatology Arthroscopy. 2016;24(8):2663-71.

34. Shin $\mathrm{K}-\mathrm{H}$, Jang K-M, Han S-B. Residual varus alignment can reduce joint awareness, restore joint parallelism, and preserve the soft tissue envelope during total knee arthroplasty for varus osteoarthritis. Knee Surgery Sports Traumatology Arthroscopy. 2020.

35. Behrend H, Giesinger K, Giesinger JM, Kuster MS. The "Forgotten Joint" as the Ultimate Goal in Joint Arthroplasty Validation of a New Patient-Reported Outcome Measure. Journal of Arthroplasty. 2012;27(3):430-6.

36. Gu Y, Roth JD, Howell SM, Hull ML. How Frequently Do Four Methods for Mechanically Aligning a Total Knee Arthroplasty Cause Collateral Ligament Imbalance and Change Alignment from Normal in 
White Patients? AAOS Exhibit Selection. Journal of Bone and Joint Surgery-American Volume. 2014;96A(12).

37. Howell SM, Howell SJ, Kuznik KT, Cohen J, Hull ML. Does A Kinematically Aligned Total Knee Arthroplasty Restore Function Without Failure Regardless of Alignment Category? Clinical Orthopaedics and Related Research. 2013;471(3):1000-7.

38. Howell SM, Howell SJ, Hull ML. Assessment of the Radii of the Medial and Lateral Femoral Condyles in Varus and Valgus Knees with Osteoarthritis. Journal of Bone and Joint Surgery-American Volume. 2010;92A(1):98-104.

39. Gao Z-x, Long N-j, Zhang S-y, Yu W, Dai Y-x, Xiao C. Comparison of Kinematic Alignment and Mechanical Alignment in Total Knee Arthroplasty: A Meta-analysis of Randomized Controlled Clinical Trials. Orthopaedic Surgery. 2020;12(6):1567-78.

40. Courtney PM, Lee G-C. Early Outcomes of Kinematic Alignment in Primary Total Knee Arthroplasty: A Meta-Analysis of the Literature. Journal of Arthroplasty. 2017;32(6):2028-+.

41. Takahashi T, Ansari J, Pandit HG. Kinematically Aligned Total Knee Arthroplasty or Mechanically Aligned Total Knee Arthroplasty. Journal of Knee Surgery. 2018;31(10):999-1006.

42. Oh S-M, Bin S-I, Kim J-Y, Lee B-S, Kim J-M. Impact of preoperative varus deformity on postoperative mechanical alignment and long-term results of "mechanical" aligned total knee arthroplasty. Orthopaedics \& Traumatology-Surgery \& Research. 2019;105(6):1061-6.

\section{Figures}




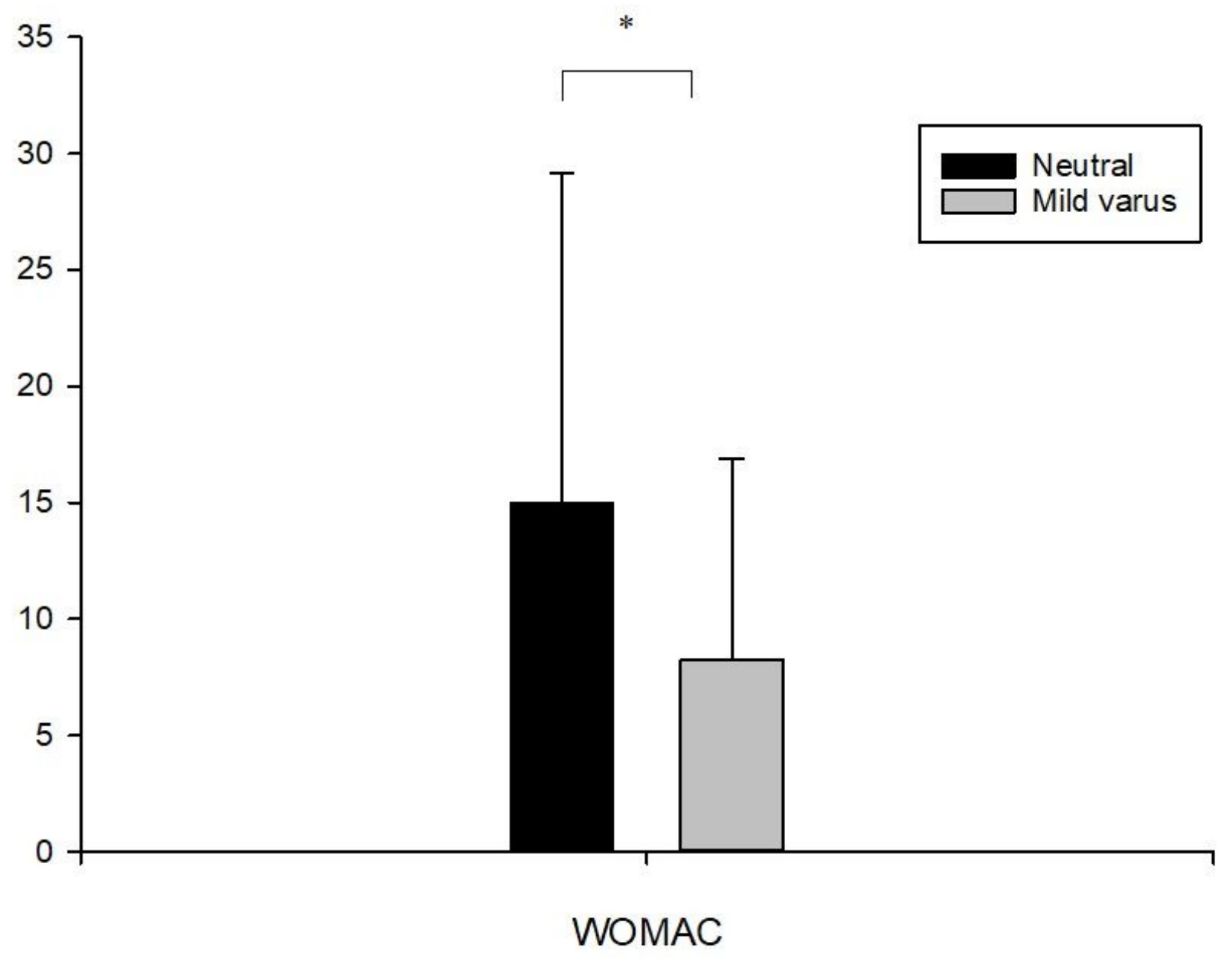

Figure 1

Differences in WOMAC between patients with neutral and mild varus $* \mathrm{P}<0.05$. Abbreviations: WOMAC: Western Ontario and McMaster Universities Osteoarthritis Index score 


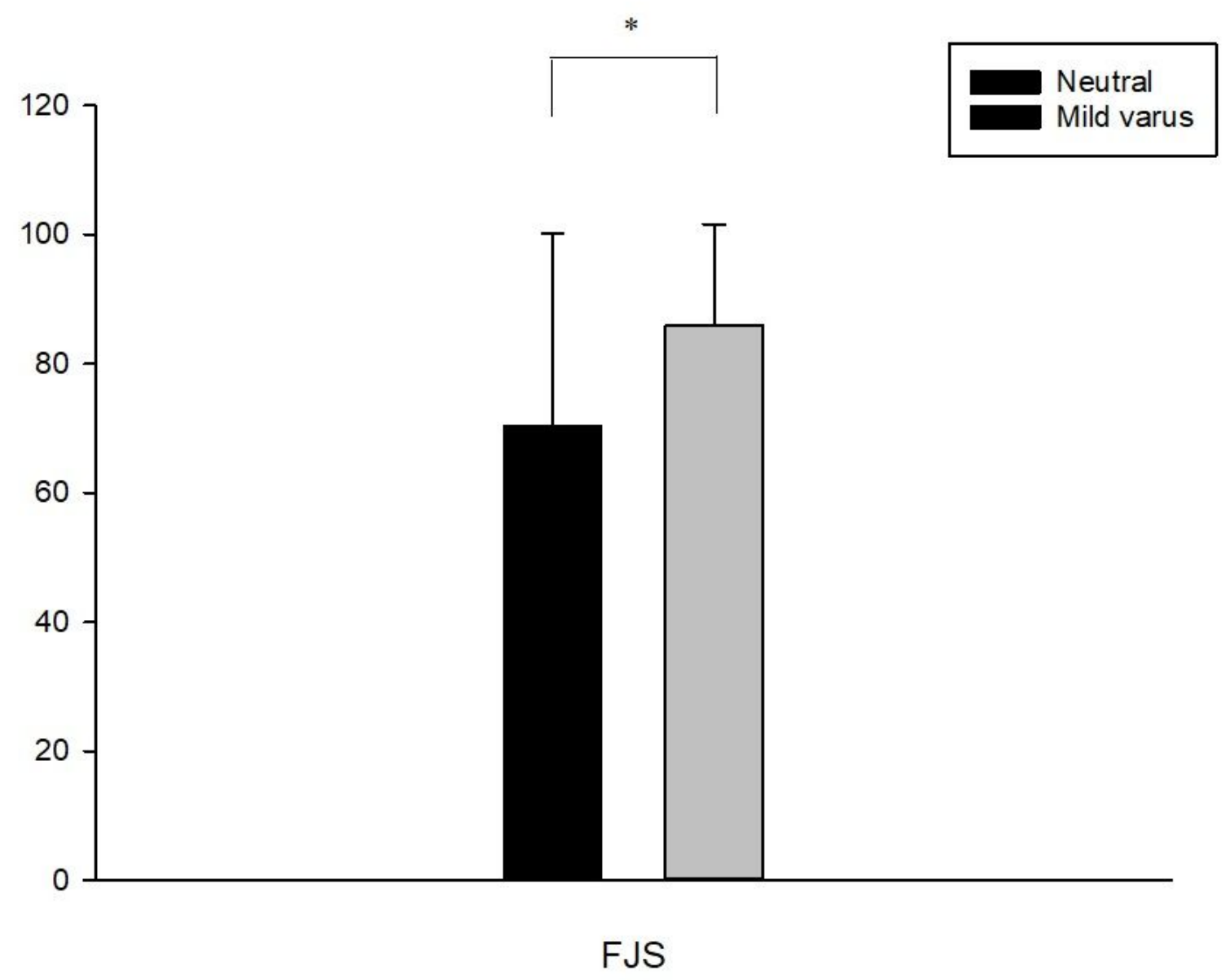

Figure 2

Differences in FJS between patients with neutral and mild varus ${ }^{*} \mathrm{P}<0.05$. Abbreviations: FJS: Forgotten Joint Score 